\title{
Espécies Potenciais para Recuperação de Áreas de Preservação Permanente no Planalto Catarinense
}

\author{
Paula Iaschitzki Ferreira ${ }^{1}$, Juliano Pereira Gomes ${ }^{1}$, Felipe Batista ${ }^{1}$, \\ Alison Paulo Bernardi², Newton Clóvis Freitas da Costa ${ }^{2}$, \\ Roseli Lopes da Costa Bortoluzzi ${ }^{1,3}$, Adelar Mantovani ${ }^{1,3}$ \\ 'Programa de Pós-graduação em Produção Vegetal, Centro de Ciências Agroveterinárias - CAV, \\ Universidade do Estado de Santa Catarina - UDESC, Lages/SC, Brasil \\ ${ }^{2}$ Centro de Ciências Agroveterinárias - CAV, Universidade do Estado de Santa Catarina - UDESC, Lages/SC, Brasil \\ ${ }^{3}$ Departamento de Engenharia Florestal, Centro de Ciências Agroveterinárias - CAV, \\ Universidade do Estado de Santa Catarina - UDESC, Lages/SC, Brasil
}

\begin{abstract}
RESUMO
Com o objetivo de selecionar espécies para programas de recuperação em Áreas de Preservação Permanente no Planalto Catarinense foi realizado o levantamento da composição florística e da estrutura fitossociológica e, a caracterização dos grupos ecológicos e síndromes de dispersão das espécies ocorrentes nestes locais. A área de estudo, fazenda Campo de Dentro, localizase no município de Otacílio Costa (SC), onde foi empregado o método de quadrantes, em 20 transecções compostas por 20 pontos amostrais. As espécies que apresentaram os maiores valores de importância, potenciais para recuperação, foram Sebastiania commersoniana (Baill.) L.B.Sm. \& Downs; Mimosa scabrella Benth.; Cinnamomum amoenum (Nees \& Mart.) Kosterm.; Lithraea brasiliensis Marchand, Ocotea pulchella (Nees \& Mart.) Mez; Ilex paraguariensis A.St.Hil.; Matayba elaeagnoides Radlk.; Ilex theezans Mart. exReissek e Vernonanthura discolor (Spreng.) H.Rob., que representam os diferentes grupos ecológicos, destacando-se a zoocoria como síndrome de dispersão. A alta diversidade de espécies arbóreas evidencia a riqueza desses locais que, muitas vezes, são negligenciados em projetos de recuperação.
\end{abstract}

Palavras-chave: Floresta Ombrófila Mista, composição florística, grupo ecológico.

\section{Potential Species for Recovery of Permanent Preservation Areas in the highlands of Santa Catarina state, Brazil}

\begin{abstract}
In this study, we carried out a survey of the floristic composition, phytosociological structure, ecological groups, and dispersal syndromes of the species occurring in riparian zones in the highlands of Santa Catarina state in order to select species for recovery programs. The study area is located in the municipality of Otacílio Costa. We employed the point-centered quadrant method allocated in twenty transects with twenty points each. The following species presented the highest importance values and recovery potentials: Sebastiania commersoniana (Baill.) L.B.S.m. \& Downs, Mimosa scabrella Benth., Cinnamomum amoenum (Nees \& Mart.) Kosterm., Lithraea brasiliensis Marchand, Ocotea pulchella (Nees \& Mart.) Mez, Ilex paraguariensis A.St.Hil., Matayba elaeagnoides Raldk., Ilex theezans Mart. ex Reissek and Vernonanthura discolor (Spreng.) H.Rob. These species represent different ecological groups, and zoochory is the most important dispersion syndrome. The high diversity of tree species shows the richness of these locations, which are often overlooked in recovery projects.
\end{abstract}

Keywords: Araucaria forest, floristic composition, environmental group. 


\section{INTRODUÇÃO}

A fragmentação é um processo antrópico que causa descontinuidade das unidades de uma paisagem, resultando em mudanças na composição e na diversificação das comunidades que nela habitam (Rodrigues et al., 2010). Diversos fenômenos são gerados em função desta ação, como isolamento e redução das áreas propícias à sobrevivência de populações, extinções locais, redução da variabilidade genética e, consequentemente, culminando-se com a perda de biodiversidade (Metzger, 1999).

O estado de Santa Catarina tem todo seu território inserido no bioma Mata Atlântica, composto pelas formações de Floresta Ombrófila Densa, Floresta Ombrófila Mista e Floresta Estacional Decidual (IBGE, 1992). O Planalto Catarinense pertence à tipologia Floresta Ombrófila Mista ou como também é chamada, Mata ou Floresta com Araucária. As florestas com araucária encontram-se altamente reduzidas entre 1 a $2 \%$ de sua área original, devido à intensa exploração no primeiro ciclo econômico ocorrido no Sul do Brasil, e atualmente seus remanescentes são protegidos por lei devido a importância ambiental e científica desta formação (Rode et al., 2011).

A legislação brasileira estabelece, desde 1965, uma faixa marginal mínima de cinco metros de mata ciliar ao longo de cursos d'água; tal faixa, que passou para $30 \mathrm{~m}$, com alterações na legislação em 1989, deve ser preservada sob a forma de Áreas de Preservação Permanente (APPs), embora, em muitos locais, a proteção não tenha ocorrido. Por outro lado, atualmente, há um crescente movimento com o objetivo de conservar e recuperar os ambientes florestais, que tem sido motivado principalmente pela aplicação de legislações e pressões dos mercados internacionais.

Segundo Neri et al. (2011) escolher corretamente a comunidade de plantas que irá iniciar o processo de sucessão em uma área degradada é um dos pontos críticos do processo de recuperação. Estudos fitossociológicos de ambientes naturais preservados e alterados têm como propósito não apenas a escolha de espécies, mas também descobrir como empregálas eficientemente nos projetos de recomposição e recuperação (Ferretti, 2002; Attanasio, 2008; Neri et al., 2011).

Este trabalho teve como objetivo selecionar espécies potenciais para recuperação de Áreas de Preservação Permanente de Floresta Ombrófila Mista, por meio do levantamento da composição florística e da estrutura fitossociológica, assim como caracterizar a síndrome de dispersão e o grupo ecológico de espécies arbóreas, a fim de contribuir para a formação de uma base de dados florísticoestruturais de ambientes inseridos no Planalto Catarinense.

\section{MATERIAL E MÉTODOS}

A fazenda Campo de Dentro (sede $27^{\circ} 39^{\prime} 15^{\prime \prime} \mathrm{S}$ e $49^{\circ} 48^{\prime} 26^{\prime \prime} \mathrm{W}$ ) está localizada no município de Otacílio Costa (SC), a aproximadamente $850 \mathrm{~m}$ de altitude. A precipitação média é de $1.700 \mathrm{~mm} / \mathrm{ano} \mathrm{e}$ a temperatura média anual varia em torno de $17^{\circ} \mathrm{C}$, sendo o clima do tipo Cfb, conforme classificação de Köeppen (1948), apresentando geadas frequentes, em média 15 por ano (Motta et al., 1971).

A fazenda possui área total de 2.500 hectares, que era composta originalmente por campos associados a fragmentos de Floresta Ombrófila Mista; esta, em meados de 1970, teve boa parte da sua área substituída por reflorestamentos de espécies exóticas, principalmente do gênero Pinus. Após dois ciclos de corte do Pinus spp., permaneceram 330,67 hectares de floresta nativa remanescente, conservados como Áreas de Preservação Permanente (APPs), associados a cursos d'água e distribuídos na forma de manchas e estreitos corredores, favorecidos pela intrincada rede hidrográfica da fazenda. Estas são as áreas objeto do presente estudo. No momento da implantação do terceiro ciclo, aproximadamente 232 hectares, que se enquadram na categoria de APPs e que estavam sendo utilizados para fins produtivos, foram deixados sob ação da regeneração natural da vegetação, sendo estas áreas denominadas Áreas em Recuperação Ambiental (ARA) (Figura 1).

Para a realização do levantamento da composição florística e da estrutura fitossociológica do componente arbóreo, foi empregado o método de quadrantes (Cottam \& Curtis, 1956). Os pontos quadrantes foram instalados ao longo de transecções 


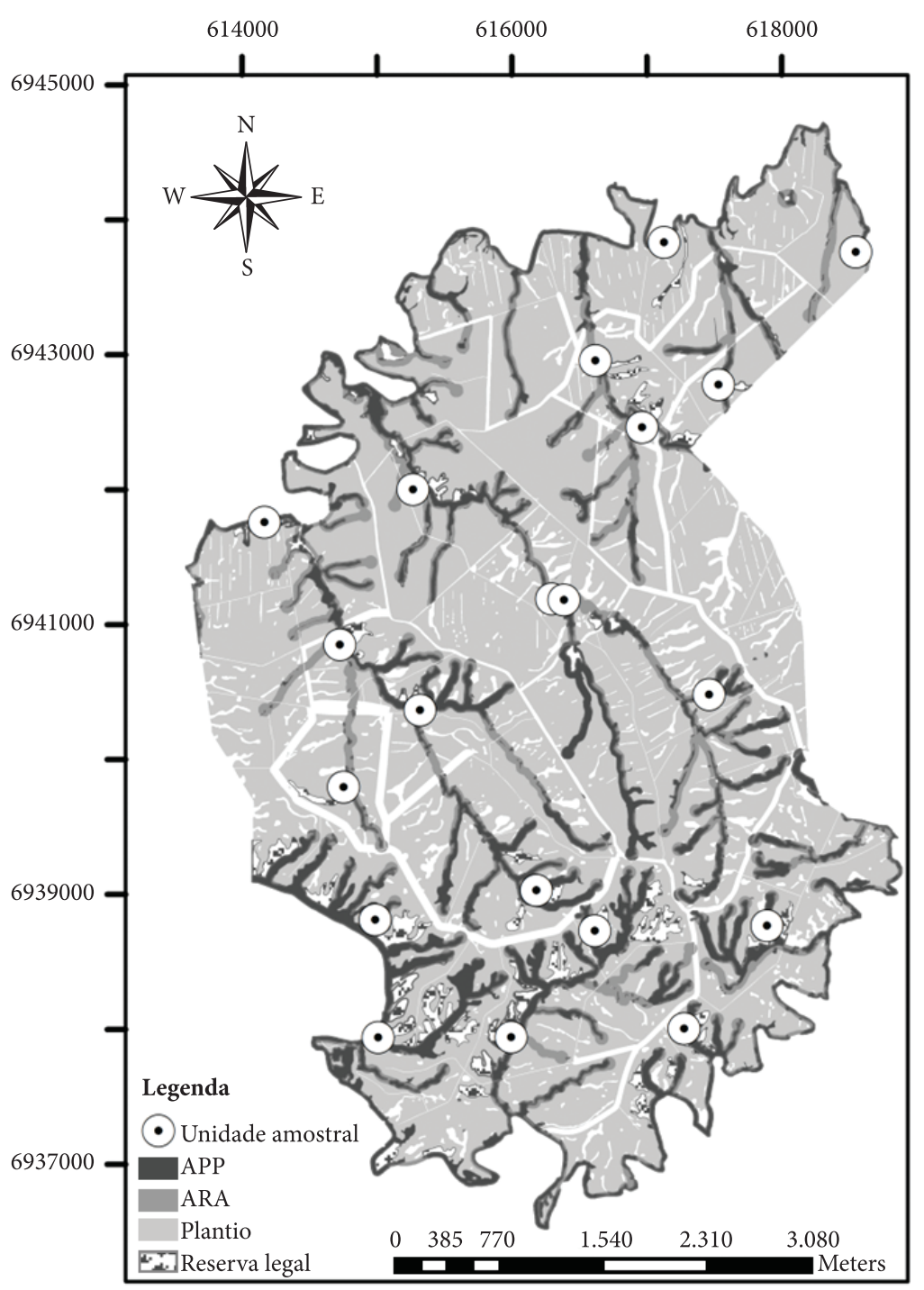

Figura 1. Mapa de uso do solo da fazenda Campo de Dentro, Otacílio Costa-SC, e distribuição das transecções. $\mathrm{APP}=$ Área de Preservação Permanente; ARA = Área em Recuperação Ambiental.

Figure 1. Map of land use of Campo de Dentro farm, Otacílio Costa (SC) and the sampling units distribution. $\mathrm{APP}=$ Permanent Preservation Area; ARA = Environmental Recovery Area.

dentro das APPs, limitadas pelos plantios ou pelas ARAs, dispostas de forma a abranger toda a área da fazenda (Figura 1). Por se tratar de áreas íngremes os pontos foram instalados do terço superior para o inferior das encostas e vice-versa, até ser alcançado o número de 20 pontos, com distância entre pontos de $15 \mathrm{~m}$, totalizando uma transecção de $300 \mathrm{~m}$ em cada fragmento (APP). Em cada ponto quadrante foi registrado o indivíduo arbóreo mais próximo do ponto central, que apresentasse diâmetro à altura do peito $(\mathrm{DAP}) \geq 5 \mathrm{~cm}$, sendo mensurados o DAP e a distância da base do tronco de cada indivíduo ao ponto central de amostragem.

Os indivíduos amostrados foram identificados em campo, quando possível, e aqueles não identificados in loco foram coletados, herborizados e identificados com o auxílio de literatura especializada, sendo os nomes científicos baseados na Flora do Brasil (Forzza et al., 2010) e na Flora Digital do Rio Grande do Sul (FDRS, 2011). Os materiais coletados férteis estão depositados no Herbário LUSC (Herbário Lages da Universidade do Estado de Santa Catarina), com sede em Lages-SC. 
Foram calculados os seguintes descritores fitossociológicos: densidade relativa, frequência relativa, dominância relativa e índice de valor de importância, de acordo com Martins (1993). $\mathrm{Na}$ apresentação dos resultados, o valor de importância foi dividido por três, como sugerido por Holdridge et al. (1971), para facilitar sua interpretação, de maneira que o valor possa representar uma porcentagem.

Para a caracterização das espécies quanto aos grupos ecológicos e síndromes de dispersão, foram consultados diversos autores que se utilizaram destes dados em trabalhos abrangendo florestas do domínio da Mata Atlântica, especialmente consultas à Flora Ilustrada Catarinense (Reitz, 1971). Segue a nomenclatura adotada para os grupos ecológicos: pioneiras $(\mathrm{Pi})$, secundárias iniciais $(\mathrm{Si})$ e secundárias tardias (St) (Gandolfi et al., 1995); para as síndromes de dispersão, zoocóricas (Zoo), anemocóricas (Ane) e autocóricas (Auto) (Van der Pijl, 1982).

\section{RESULTADOS E DISCUSSÃO}

No total, foram instalados 400 pontos, que perfizeram uma área de 1,69 hectare, onde foram mensurados 1.600 indivíduos, pertencentes a 97 espécies distribuídas em 30 famílias (Tabela 1). Quanto à classificação botânica das espécies amostradas, duas pertencem a pteridófitas (Cyatheaceae e Dicksoniaceae), uma gimnosperma (Araucariaceae) e as demais, angiospermas. As famílias que apresentaram maior riqueza foram Myrtaceae (23), Asteraceae (11), Lauraceae (10) e Solanaceae (9), conforme Figura 2. Myrtaceae também apresentou o maior número de indivíduos (293), seguida de Dicksoniaceae (275), sendo esta representada por uma única espécie (Dicksonia sellowiana Hook.), Euphorbiaceae (183) e Fabaceae (145) (Figura 3). As famílias de maior riqueza contribuíram com 52,47\% do total das espécies encontradas nas Áreas de Preservação Permanente (APPs) da fazenda. Klein (1984) já fazia referência à importância de Myrtaceae na Floresta Ombrófila Mista (FOM), pelo número de indivíduos encontrados. Esta importância vem sendo comprovada em diversos trabalhos realizados nas Regiões Sul e Sudeste, nos últimos anos (Jarenkow \& Waechter, 2001; Bianchini et al., 2003; França \&
Stehmann, 2004; Barddal et al., 2004; Sonego et al., 2007; Higuchi et al., 2012).

As espécies que apresentaram os maiores índices de valor de importância (IVI) foram: Dicksonia sellowiana; Sebastiania commersoniana (Baill.) L.B.Sm. \& Downs; Mimosa scabrella Benth.; Cinnamomum amoenum (Nees \& Mart.) Kosterm.; Lithraea brasiliensis Marchand; Ocotea pulchella (Nees \& Mart.) Mez; Ilex paraguariensis A.St.-Hil.; Matayba elaeagnoides Radlk.; Ilex theezans Mart. ex

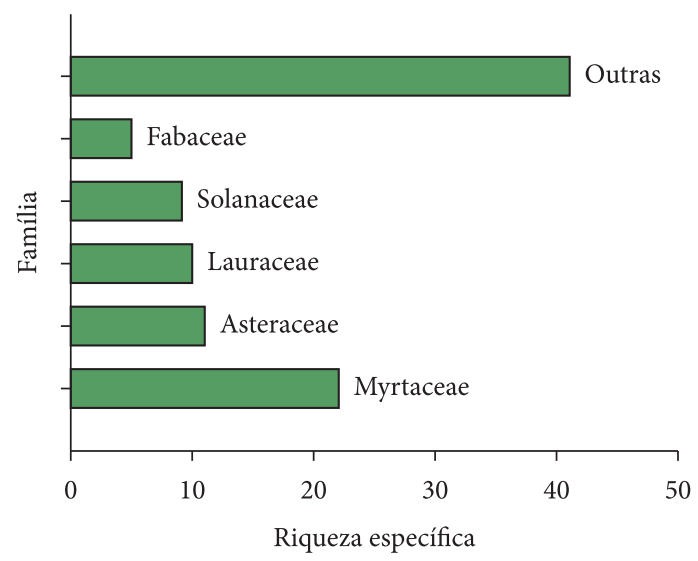

Figura 2. Riqueza de espécies por família em Áreas de Preservação Permanente da fazenda Campo de Dentro, Otacílio Costa (SC), 2011.

Figure 2. Species richness per family in Permanent Preservation Areas of the Campo de Dentro Farm, Otacílio Costa (SC), 2011.

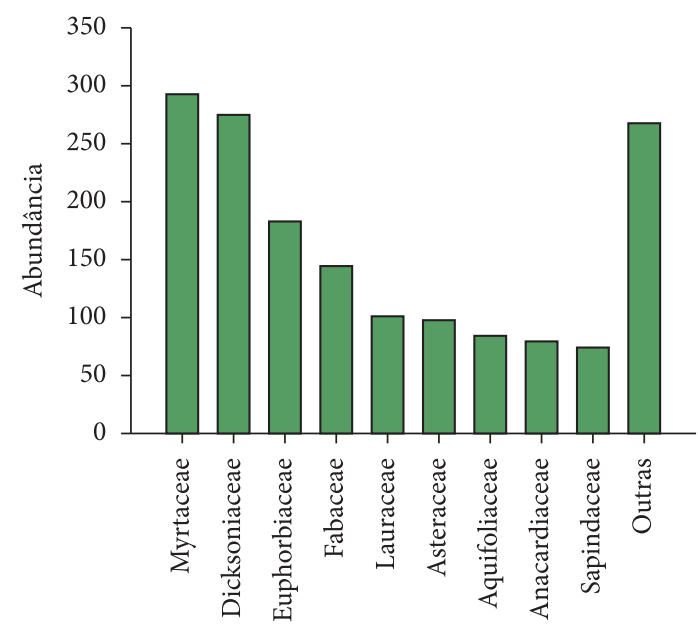

Figura 3. Número de indivíduos (abundância) por família em Áreas de Preservação Permanente da fazenda Campo de Dentro, Otacílio Costa-SC, 2011.

Figure 3. Number of individuals (abundance) per Family in Permanent Preservation Areas of the Campo de Dentro Farm, Otacílio Costa (SC), 2011. 
Tabela 1. Parâmetros fitossociológicos das espécies amostradas nas Áreas de Preservação Permanente da fazenda Campo de Dentro, Otacílio Costa-SC, ordenadas em ordem decrescente do índice de valor de importância. $\mathrm{DR}$ = densidade relativa; $\mathrm{FR}$ = frequência relativa; $\mathrm{DoR}=$ dominância relativa; $\mathrm{IVI}=$ índice de valor de importância; $\mathrm{SD}=$ Síndrome de dispersão; GE = Grupo ecológico; Ind. = indeterminado.

Table 1. Phytosociology parameters of the species at the stand of the Permanente Preservation Areas off the Campo de Dentro farm, Otacílio Costa (SC), ordered en decreasing IVI. DR = relative density; FR = relative frequency; $\mathrm{DoR}$ = relative dominance; IVI = importance value index; GE = ecological group; $\mathrm{SD}=$ dispersion syndrome; Ind. = indeterminate.

\begin{tabular}{|c|c|c|c|c|c|c|c|}
\hline Família & Espécie & DR & FR & DoR & IVI & SD & GE \\
\hline Dicksoniaceae & Dicksonia sellowiana Hook. & 17,19 & 12,72 & 19,57 & 16,49 & Ind. & Ind. \\
\hline Euphorbiaceae & Sebastiania commersoniana (Baill.) L.B.Sm. \& Downs & 11,13 & 7,43 & 6,19 & 8,25 & Ane & $\mathrm{Si}$ \\
\hline Fabaceae & Mimosa scabrella Benth. & 6,44 & 4,54 & 9,27 & 6,75 & Auto & $\mathrm{Pi}$ \\
\hline Lauraceae & Cinnamomum amoenum (Nees \& Mart.) Kosterm. & 2,25 & 2,73 & 8,15 & 4,38 & Zoo & St \\
\hline Anacardiaceae & Lithraea brasiliensis Marchand & 4,19 & 4,29 & 4,14 & 4,21 & Zoo & $\mathrm{Pi}$ \\
\hline Lauraceae & Ocotea pulchella (Nees \& Mart.) Mez & 1,69 & 2,23 & 5,91 & 3,28 & Zoo & $\mathrm{Si}$ \\
\hline Aquifoliaceae & Ilex paraguariensis A.St.-Hil. & 2,63 & 3,06 & 3,01 & 2,90 & Zoo & St \\
\hline Sapindaceae & Matayba elaeagnoides Radlk. & 2,44 & 2,64 & 3,29 & 2,79 & Zoo & St \\
\hline Aquifoliaceae & Ilex theezans Mart. ex Reissek & 2,19 & 2,64 & 3,21 & 2,68 & Zoo & $\mathrm{Si}$ \\
\hline Asteraceae & Vernonanthura discolor (Spreng.) H.Rob. & 3,06 & 2,97 & 1,79 & 2,61 & Ane & $\mathrm{Pi}$ \\
\hline Myrtaceae & Myrceugenia euosma (O.Berg) D.Legrand & 2,75 & 2,89 & 1,94 & 2,53 & Zoo & $\mathrm{Si}$ \\
\hline Clethraceae & Clethra scabra Pers. & 2,13 & 2,56 & 2,49 & 2,39 & Auto & $\mathrm{Pi}$ \\
\hline Myrtaceae & Calyptranthes concinna DC. & 2,44 & 3,06 & 1,24 & 2,24 & Zoo & St \\
\hline Araucariaceae & Araucaria angustifolia (Bertol.) Kuntze & 1,81 & 2,31 & 2,41 & 2,18 & Zoo & $\mathrm{Pi}$ \\
\hline Myrtaceae & Myrcia palustris DC. & 1,69 & 1,98 & 1,48 & 1,72 & Zoo & $\mathrm{Pi}$ \\
\hline Myrtaceae & Blepharocalyx salicifolius (Kunth) O.Berg & 1,88 & 2,23 & 0,97 & 1,69 & Zoo & $\mathrm{Si}$ \\
\hline Asteraceae & Piptocarpha angustifolia Dusénex Malme & 2,00 & 1,65 & 1,03 & 1,56 & Ane & $\mathrm{Pi}$ \\
\hline Lauraceae & Ocotea puberula (Rich.) Nees & 0,94 & 1,07 & 2,60 & 1,54 & Zoo & $\mathrm{Si}$ \\
\hline Myrtaceae & Myrceugenia myrcioides (Cambess.) O.Berg & 2,00 & 1,57 & 1,01 & 1,52 & Zoo & St \\
\hline Myrtaceae & Myrceugenia glaucescens (Cambess.) D.Legrand \& Kausel & 2,00 & 1,57 & 1,01 & 1,52 & Zoo & St \\
\hline Fabaceae & Inga lentiscifolia Benth. & 1,75 & 2,06 & 0,55 & 1,46 & Zoo & St \\
\hline Meliaceae & Cedrela fissilis Vell. & 0,50 & 0,66 & 2,83 & 1,33 & Ane & $\mathrm{Pi}$ \\
\hline Myrtaceae & Eugenia catharinae O. Berg & 1,38 & 1,57 & 0,70 & 1,22 & Zoo & $\mathrm{Si}$ \\
\hline Styracaceae & Styrax leprosus Hook. \& Arn. & 1,44 & 1,65 & 0,55 & 1,21 & Zoo & $\mathrm{Si}$ \\
\hline Salicaceae & Casearia decandra Jacq. & 1,56 & 1,65 & 0,36 & 1,19 & Zoo & St \\
\hline Sapindaceae & Allophylus guaraniticus (A. St.-Hil.) Radlk. & 1,25 & 1,40 & 0,80 & 1,15 & Zoo & St \\
\hline Sapindaceae & Allophylus edulis (A.St.-Hil. et al.) Hieron. ex Niederl. & 1,00 & 1,07 & 0,86 & 0,98 & Zoo & $\mathrm{Si}$ \\
\hline Myrtaceae & Myrcia selloi (Spreng.) N.Silveira & 1,06 & 1,32 & 0,31 & 0,90 & Zoo & $\mathrm{Si}$ \\
\hline Myrsinaceae & Myrsine coriacea (Sw.) R.Br. ex Roem. \&Schult. & 0,81 & 1,07 & 0,54 & 0,81 & Zoo & $\mathrm{Si}$ \\
\hline Anacardiaceae & Schinus terebinthifolius Raddi & 0,75 & 0,91 & 0,55 & 0,74 & Zoo & $\mathrm{Pi}$ \\
\hline Cunoniaceae & Lamanonia ternata Vell. & 0,50 & 0,66 & 1,05 & 0,74 & Ane & $\mathrm{Pi}$ \\
\hline Cardiopteridaceae & Citronella paniculata (Mart.) R.A.Howard & 0,69 & 0,91 & 0,52 & 0,71 & Zoo & St \\
\hline Lauraceae & Nectandra grandiflora Nees & 0,56 & 0,66 & 0,78 & 0,67 & Zoo & St \\
\hline Elaeocarpaceae & Sloanea hirsuta (Schott) Planch. ex Benth. & 0,19 & 0,25 & 1,49 & 0,64 & Zoo & St \\
\hline Myrtaceae & Campomanesia xanthocarpa Berg & 0,50 & 0,58 & 0,60 & 0,56 & Zoo & $\mathrm{Si}$ \\
\hline Myrtaceae & Myrcia splendens (Sw.) DC. & 0,69 & 0,83 & 0,12 & 0,55 & Zoo & $\mathrm{Si}$ \\
\hline Winteraceae & Drimys brasiliensis Miers & 0,44 & 0,58 & 0,50 & 0,51 & Zoo & St \\
\hline Symplocaceae & Symplocos tetrandra Mart. & 0,50 & 0,66 & 0,35 & 0,50 & Zoo & St \\
\hline Solanaceae & Solanum mauritianum Scop. & 0,75 & 0,66 & 0,08 & 0,50 & Zoo & $\mathrm{Pi}$ \\
\hline Aquifoliaceae & Ilex dumosa Reissek & 0,44 & 0,58 & 0,48 & 0,50 & Zoo & $\mathrm{Pi}$ \\
\hline Bignoniaceae & Jacaranda puberula Cham. & 0,50 & 0,58 & 0,28 & 0,45 & Ane & $\mathrm{Si}$ \\
\hline Annonaceae & Annona rugulosa (Schltdl.) H.Rainer & 0,50 & 0,66 & 0,19 & 0,45 & Zoo & $\mathrm{Si}$ \\
\hline Fabaceae & Inga vera Willd. & 0,44 & 0,58 & 0,28 & 0,43 & Zoo & $\mathrm{Si}$ \\
\hline Fabaceae & Inga virescens Benth. & 0,38 & 0,50 & 0,40 & 0,43 & Zoo & $\mathrm{Si}$ \\
\hline Lauraceae & Nectandra megapotamica (Spreng.) Mez & 0,38 & 0,50 & 0,40 & 0,42 & Zoo & St \\
\hline Myrtaceae & Myrceugenia oxysepala (Burret) D. Legrand \& Kausel & 0,31 & 0,41 & 0,47 & 0,40 & Zoo & St \\
\hline Cyatheaceae & Alsophila setosa Kaulf. & 0,44 & 0,50 & 0,22 & 0,39 & Ind. & Ind. \\
\hline
\end{tabular}


Tabela 1. Continuação...

\begin{tabular}{|c|c|c|c|c|c|c|c|}
\hline Família & Espécie & DR & FR & DoR & IVI & SD & GE \\
\hline Salicaceae & Casearia obliqua Spreng. & 0,44 & 0,58 & 0,14 & 0,38 & Zoo & St \\
\hline Rutaceae & Zanthoxylum rhoifolium Lam. & 0,38 & 0,41 & 0,29 & 0,36 & Zoo & $\mathrm{Pi}$ \\
\hline Symplocaceae & Symplocos tenuifolia Brand & 0,44 & 0,58 & 0,05 & 0,35 & Zoo & $\mathrm{Pi}$ \\
\hline Asteraceae & Baccharis subdentata DC. & 0,31 & 0,41 & 0,30 & 0,34 & Ane & $\mathrm{Pi}$ \\
\hline Symplocaceae & Symplocos uniflora (Pohl) Benth. & 0,31 & 0,41 & 0,23 & 0,32 & Zoo & St \\
\hline Myrtaceae & Eugenia burkartiana (D.Legrand) D.Legrand & 0,38 & 0,50 & 0,04 & 0,30 & Zoo & $\mathrm{Si}$ \\
\hline Euphorbiaceae & Actinostemon concolor (Spreng.) Müll. Arg. & 0,19 & 0,25 & 0,45 & 0,30 & Auto & St \\
\hline Myrtaceae & Myrrhinium atropurpureum Schott & 0,31 & 0,41 & 0,08 & 0,27 & Zoo & St \\
\hline Myrtaceae & Myrceugenia miersiana (Gardner) D.Legrand \& Kausel & 0,31 & 0,41 & 0,06 & 0,26 & Zoo & $\mathrm{Si}$ \\
\hline Solanaceae & Capsicum villosum Sendtn. & 0,31 & 0,41 & 0,05 & 0,26 & Zoo & $\mathrm{Si}$ \\
\hline Myrtaceae & Myrcia guianensis (Aubl.) DC. & 0,31 & 0,33 & 0,10 & 0,25 & Zoo & $\mathrm{Si}$ \\
\hline Solanaceae & Solanum pseudoquina A.St.-Hil. & 0,25 & 0,33 & 0,14 & 0,24 & Zoo & $\mathrm{Si}$ \\
\hline Rutaceae & Zanthoxylum kleinii (R.S.Cowan) P.G.Waterman & 0,25 & 0,33 & 0,11 & 0,23 & Zoo & $\mathrm{Pi}$ \\
\hline Proteaceae & Roupala montana (Klotzsch) K.S.Edwards & 0,19 & 0,25 & 0,17 & 0,20 & Zoo & St \\
\hline Lauraceae & Persea major (Meisn.) L.E.Kopp & 0,19 & 0,25 & 0,12 & 0,19 & Zoo & St \\
\hline Lauraceae & Ocotea porosa (Nees \& Mart.) Barroso & 0,19 & 0,25 & 0,10 & 0,18 & Zoo & St \\
\hline Myrtaceae & Prunus myrtifolia (L.) Urb. & 0,19 & 0,25 & 0,05 & 0,16 & Zoo & $\mathrm{Si}$ \\
\hline Asteraceae & Eupatorium sp. & 0,19 & 0,25 & 0,02 & 0,15 & Ane & $\mathrm{Pi}$ \\
\hline Bignoniaceae & Handroanthus albus (Cham.) Mattos & 0,13 & 0,17 & 0,14 & 0,15 & Ane & $\mathrm{Pi}$ \\
\hline Myrtaceae & Myrcia hartwegiana (O. Berg) Kiaersk. & 0,19 & 0,17 & 0,08 & 0,14 & Zoo & $\mathrm{Si}$ \\
\hline Myrsinaceae & Myrsine umbellata Mart. & 0,13 & 0,17 & 0,07 & 0,12 & Zoo & $\mathrm{Pi}$ \\
\hline Myrsinaceae & Myrsine parvula (Mez) Otegui. & 0,13 & 0,17 & 0,05 & 0,11 & Zoo & $\mathrm{Si}$ \\
\hline Moraceae & Ficus cf. luschnathiana (Miq.) Miq. & 0,13 & 0,17 & 0,02 & 0,10 & Zoo & St \\
\hline Solanaceae & Solanum paranense Dusén & 0,13 & 0,17 & 0,02 & 0,10 & Zoo & $\mathrm{Si}$ \\
\hline Asteraceae & Baccharis oblongifolia DC. & 0,13 & 0,17 & 0,01 & 0,10 & Ane & $\mathrm{Pi}$ \\
\hline Fabaceae & Inga striata Benth. & 0,13 & 0,08 & 0,13 & 0,09 & Zoo & $\mathrm{Si}$ \\
\hline Solanaceae & Solanum ramulosum Sendtn. & 0,06 & 0,08 & 0,11 & 0,09 & Zoo & $\mathrm{Si}$ \\
\hline Solanaceae & Sp 1 & 0,06 & 0,08 & 0,08 & 0,08 & Ind. & Ind. \\
\hline Myrtaceae & Myrciaria delicatula (DC.) O.Berg & 0,06 & 0,08 & 0,02 & 0,07 & Zoo & St \\
\hline Lauraceae & Aniba firmula (Nees \& Mart.) Mez & 0,06 & 0,08 & 0,06 & 0,07 & Zoo & St \\
\hline Euphorbiaceae & Sapium glandulosum (L.) Morong & 0,06 & 0,08 & 0,03 & 0,06 & Auto & $\mathrm{Pi}$ \\
\hline Solanaceae & Solanum compressum L.B.Sm. \& Downs & 0,06 & 0,08 & 0,03 & 0,06 & Zoo & $\mathrm{Si}$ \\
\hline Myrtaceae & Eugenia neomyrtifolia Sobral & 0,06 & 0,08 & 0,03 & 0,06 & Zoo & $\mathrm{Si}$ \\
\hline Myrtaceae & Myrceugenia ovata (Hook. \& Arn.) O.Berg & 0,06 & 0,08 & 0,03 & 0,06 & Zoo & St \\
\hline Myrtaceae & Eugenia uniflora L. & 0,06 & 0,08 & 0,02 & 0,06 & Zoo & $\mathrm{Si}$ \\
\hline Asteraceae & Gochnatia polymorpha (Less.) Cabrera & 0,06 & 0,08 & 0,02 & 0,05 & Ane & $\mathrm{Pi}$ \\
\hline Solanaceae & Solanum sp. & 0,06 & 0,08 & 0,02 & 0,05 & Zoo & $\mathrm{Pi}$ \\
\hline Asteraceae & Dasyphyllum brasiliense (Spreng.) Cabrera & 0,06 & 0,08 & 0,01 & 0,05 & Ane & $\mathrm{Si}$ \\
\hline Myrtaceae & Myrciaria tenella (DC.) O.Berg & 0,06 & 0,08 & 0,01 & 0,05 & Zoo & St \\
\hline Euphorbiaceae & Sebastiania brasiliensis Spreng. & 0,06 & 0,08 & 0,01 & 0,05 & Auto & $\mathrm{Si}$ \\
\hline Asteraceae & Baccharis oxyodonta DC. & 0,06 & 0,08 & 0,01 & 0,05 & Ane & $\mathrm{Pi}$ \\
\hline Melastomataceae & Miconia cinerascens Miq. & 0,06 & 0,08 & 0,01 & 0,05 & Zoo & $\mathrm{Si}$ \\
\hline Picramniaceae & Picramnia parvifolia Engl. & 0,06 & 0,08 & 0,01 & 0,05 & Zoo & St \\
\hline Salicaceae & Xylosma ciliatifolia(Clos) Eichler & 0,06 & 0,08 & 0,00 & 0,05 & Zoo & $\mathrm{Si}$ \\
\hline Lauraceae & Cinnamomum glaziovii (Mez) Kosterm. & 0,06 & 0,08 & 0,00 & 0,05 & Zoo & St \\
\hline Solanaceae & Solanum cassioides L.B.Sm. \& Downs & 0,06 & 0,08 & 0,00 & 0,05 & Zoo & $\mathrm{Si}$ \\
\hline Asteraceae & Eupatorium lymansmith (B.L. Rob.) Steyerm. & 0,06 & 0,08 & 0,00 & 0,05 & Ane & $\mathrm{Pi}$ \\
\hline Solanaceae & Solanum lacerdae Dusén & 0,06 & 0,08 & 0,00 & 0,05 & Zoo & $\mathrm{Pi}$ \\
\hline Asteraceae & $\begin{array}{l}\text { Symphyopappus itatiayensis (Hieron.) R.M.King \& } \\
\text { H.Rob. }\end{array}$ & 0,06 & 0,08 & 0,00 & 0,05 & Ane & $\mathrm{Pi}$ \\
\hline \multirow[t]{2}{*}{ Asteraceae } & Baccharis semiserrata DC. & 0,06 & 0,08 & 0,01 & 0,05 & Ane & $\mathrm{Pi}$ \\
\hline & & 100 & 100 & 100 & 100 & & \\
\hline
\end{tabular}


Reissek; Vernonanthura discolor (Spreng.) H.Rob., representando mais de $50 \%$ do IVI total.

Considerando-se que o ambiente em estudo é de Áreas de Preservação Permanente (APPs) associadas aos cursos d'água, era esperada a abundante presença de espécies como Dicksonia sellowiana, descrita como tolerante a ambientes paludosos (Sehnem, 1978; Lorenzi, 1998; Carvalho et al., 2005). Esta espécie é de comum ocorrência nas matas da Região Sul do Brasil (Reitz et al., 1983; Dias et al., 1998; Barddal et al., 2004; Loures et al., 2007) e, assim como encontrados neste trabalho, $D$. sellowiana é registrada em baixadas ou encostas úmidas, geralmente formando maciços, em razão do acúmulo de sedimentos e umidade (Martins-Ramos et al., 2011). É importante ressaltar que $D$. sellowiana se trata de um táxon que consta na Red List of Threatened Species da IUCN, conferindo às APPs da fazenda grande importância ecológica e conservacionista.

De acordo com a análise fitossociológica, é possível observar que as espécies de maior IVI são representadas pelos diferentes grupos ecológicos propostos por Gandolfi et al. (1995).

Mimosa scabrella, Lithraea brasiliensis e Vernonanthura discolor compõem o grupo das pioneiras. $M$. scabrellae $V$. discolor são citadas como espécies comuns em áreas que foram submetidas à perturbações (Machado et al., 2006; Herrera et al., 2009). Nota-se que M. scabrella é comumente encontrada nas áreas em estágio inicial nos domínios da FOM, sendo bastante tolerante em relação às condições físicas do solo (Inoue et al., 1984), pois a sua rusticidade e o caráter heliófito proporcionam a rápida cobertura de áreas de solos alterados (Baggio, 1994). Com relação à L. brasiliensis, em estudo realizado em FOM, esta apresentou alta frequência (Rondon-Neto et al., 2002), sendo também descrita como heliófita (Durigan \& Leitão-Filho, 1995; Carvalho, 1994). Dessa forma, acredita-se que o efeito de borda, ao qual esses locais estão expostos, propiciou a expansão espacial e o desenvolvimento destas espécies, contribuindo para que as mesmas obtivessem significativos valores de importância na análise fitossociológica do presente estudo.

No que se refere às espécies representantes do grupo das secundárias iniciais, Sebastiania commersoniana, Ocotea pulchella e Ilex theezans se destacam. Sebastiania commersoniana é mencionada como espécie característica do estrato arbóreo de florestas ciliares (Gibbs \& Leitão-Filho, 1978) e ocorre quase exclusivamente nas planícies aluviais, onde, não raramente, se torna a espécie dominante (Cordeiro \& Rodrigues, 2007), formando 60 a $80 \%$ do estrato contínuo das florestas de galeria (Reitz, 1988). O. puchella, também conhecida como canela lageana, foi descrita em estudo realizado em FOM, na Floresta Nacional de São Francisco de Paula-RS, com alto valor de dominância relativa (Sonego et al., 2007). Algumas lauráceas, como as pertencentes ao gênero Ocotea, aparecem com grande frequência, inclusive em remanescentes expressivamente alterados, conforme constatado em outros trabalhos (Negrelle \& Silva, 1992; Nascimento et al., 2001; Sanquetta et al., 2002). Dentre as espécies de Aquifoliaceae, I. theezans é considerada uma das mais frequentes em FOM; esta, segundo Viani \& Vieira (2007), normalmente ocorre em áreas onde também ocorre Ilex paraguariensis.

Ilex paraguariensis, Cinnamomum amoenum e Matayba elaeagnoides compõem o grupo das secundárias tardias, registradas com os maiores IVIs no presente estudo. A erva-mate (I. paraguariensis) é caracterizada como espécie florestal umbrófila e seletiva higrófila, que cresce preferencialmente em associações de estágios mais avançados de sucessão em FOM (Carvalho, 1994). Contudo, a espécie é considerada tolerante a solos de baixa fertilidade natural, resistindo a solos degradados, sendo frequente em solos com baixo teor de nutrientes trocáveis e alto teor de alumínio (Oliveira \& Rota, 1995). C. amoenum tem sido registrada em estudos da composição florística nas formações de mata de araucária (FOM) (Martins-Ramos et al., 2011; Siminski et al., 2011; Vibrans et al., 2011; Higuchi et al., 2012), o que demonstra potencial para recomposição desta tipologia. M. elaeagnoides, conhecida popularmente como miguel-pintado ou camboatá-branco, é uma espécie comum de solos bem drenados (Durigan, 1999); entretanto, foi descrita por Narvaes et al. (2008) como espécie indicadora nos locais de maior umidade, demonstrando ser uma espécie rústica, característica esta desejável para recomposição de áreas alteradas. Lima et al. (2012), em estudo de caracterização fitossociológica da vegetação no entorno de nascentes de um 
fragmento de FOM, registraram $M$. elaeagnoides como espécie de maior IVI. É importante salientar que este grupo ecológico apresenta maiores exigências edafoclimáticas, devendo ser inserido nas etapas finais do processo de recuperação, quando os ambientes já detêm espécies pioneiras e secundárias inicias estabelecidas.

Dentre as espécies que apresentaram os maiores IVI, destaca-se a síndrome de dispersão zoocórica como a mais comum. Galetti (1995) destacou os animais dispersores de sementes como principais agentes responsáveis pela regeneração das florestas, carregando sementes das matrizes florestais para áreas do entorno, possibilitando a revegetação das mesmas com o passar do tempo. Segundo Reis \& Kageyama (2003), a presença de espécies zoocóricas em áreas degradadas aumenta as chances de recolonização por permitirem a chegada de novos propágulos. Neste sentido, a introdução de espécies zoocóricas, assim como as descritas no presente estudo, pode atuar na facilitação do processo da dinâmica sucessional, consistindo numa abordagem fundamental em programas de recuperação, uma vez que proporcionam interação com a fauna, podendo favorecer a ocorrência de fluxo biológico entre os elementos da paisagem.

\section{CONCLUSÕES}

Mimosa scabrella, Lithraea brasiliensis e Vernonanthura discolor são espécies pioneiras, características de Floresta Ombrófila Mista, que detêm caráter de rusticidade, conferindo-lhes assim atributos potenciais para que sejam utilizadas na fase inicial de programas de recuperação de áreas degradadas.

Sebastiania commersoniana, Ocotea pulchella e Ilex theezans, pertencentes ao grupo das secundárias iniciais, apresentam adaptabilidade às condições ambientais inerentes as Áreas de Preservação Permanente associadas a cursos d'água, viabilizandoas, dessa forma, em programas de enriquecimento.

Matayba elaeagnoides, Cinnamomum amoenum e Ilex paraguariensis representam o grupo das secundárias tardias nas formações de Floresta Ombrófila Mista, podendo compor as etapas finais de programas de enriquecimento, em ambientes onde as espécies pioneiras e secundárias iniciais já estão estabelecidas. Tais espécies podem contribuir para a interação com a fauna, auxiliando o restabelecimento da dinâmica e do equilíbrio dos ecossistemas.

A preservação desses ambientes surge como alternativa para o resgate e a manutenção da biodiversidade, o que vem sendo comumente pouco valorizado em programas de recuperação.

\section{AGRADECIMENTOS}

Os autores agradecem à Coordenação de Aperfeiçoamento de Pessoal de Nível Superior (CAPES), pela concessão de Bolsa de Mestrado, e à Empresa Klabin, pela disponibilização das áreas e pelo apoio financeiro.

\section{STATUS DA SUBMISSÃO}

Recebido: 19/07/2012

Aceito: 17/02/2013

Publicado: 30/06/2013

\section{AUTOR(ES) PARA CORRESPONDÊNCIA}

\section{Paula Iaschitzki Ferreira}

Programa de Pós-graduação em Produção Vegetal, Centro de Ciências Agroveterinárias - CAV, Universidade do Estado de Santa Catarina UDESC, CEP 88520-000, Lages, SC, Brasil e-mail: paulaiaschitzki@hotmail.com

\section{REFERÊNCIAS}

Attanasio CM. Manual Técnico: Restauração e monitoramento da Mata Ciliar e da Reserva Legal para a Certificação Agrícola - Conservação da Biodiversidade na Cafeicultura. Piracicaba: IMAFLORA; 2008.

Baggio AJ. Estudio sobre el sistema agroforestal tradicional de labracatinga (Mimosa scabrella Benth.) en Brasil: productividad, manejo de residuos y elaboracion de compost [tese]. Madrid: Escuela Técnica Superior de Ingenieros de Montes; 1994. 242 p.

Barddal ML, Roderjan CV, Galvão F, Curcio GR. Fitossociologia do sub-bosque de uma Floresta Ombrófila Mista Aluvial, no município de Araucária, PR. Ciência Florestal 2004; 14(1): 35-45.

Bianchini E, Popolo RS, Dias MC, Pimenta JA. Diversidade e estrutura de espécies arbóreas em área 
alagável do município de Londrina, Sul do Brasil. Acta Botanica Brasilica 2003; 17: 405-419. http://dx.doi. org/10.1590/S0102-33062003000300008

Carvalho DA, Oliveira-Filho AT, Van Den Berg E, Fontes MAL, Vilela EA, Marque JJGSM. Variações florísticas e estruturais do componente arbóreo de uma floresta ombrófila alto-montana às margens do Rio Grande, Bocaina de Minas, MG, Brasil. Acta Botanica Brasilica 2005; 19: 91-109. http://dx.doi.org/10.1590/ S0102-33062005000100010

Carvalho PER. Espécies florestais brasileiras: recomendações silviculturais, potencialidades e uso da madeira. Colombo: EMBRAPA-CNPF; Brasília: EMBRAPA-SPI; 1994.

Cordeiro J, Rodrigues WA. Caracterização fitossociológica de um remanescente de Floresta Ombrófila mista em Guarapuava, PR. Revista Árvore 2007; 31(3): 545-554. http://dx.doi.org/10.1590/ S0100-67622007000300020

Cottam G, Curtis JT. The use of distance measures in phytosociological sampling. Ecology 1956;(37): 451-460. http://dx.doi.org/10.2307/1930167

Dias MC, Vieira AOS, Nakajima JN, Pimenta JA, Lobo PC. Composição florística e fitossociológica do componente arbóreo das florestas ciliares do rio Iapó, na bacia do rio Tibagi, Tibagi, PR. Revista Brasileira de Botânica 1998; 21(2): 183-195. http://dx.doi. org/10.1590/S0100-84041998000200011

Durigan ME. Florística, dinâmica e análise protéica de uma floresta ombrófila mista em São João do Triunfo - $P R$ [dissertação]. Curitiba: Universidade Federal do Paraná; 1999. 125 p.

Durigan G, Leitão-Filho HF. Florística e fitossociologia de matas ciliares do Oeste Paulista. Revista do Instituto Florestal 1995; 7(2): 197-239.

Flora Digital do Rio Grande do Sul - FDRS. Porto Alegre; 2011. [cited 2011 maio 16]. Available from: http://www6.ufrgs.br/fitoecologia/florars.

Forzza RC, Stehmann JR, Nadruz M, Filardi FLR, Costa A, Carvalho-Junior AA et al. Lista de Espécies da Flora do Brasil. Rio de Janeiro: Jardim Botânico do Rio de Janeiro; 2010 .

França GS, Stehmann JR. Composição florística e estrutura do componente arbóreo de uma floresta altimontana no município de Camanducaia, Minas Gerais, Brasil. Revista Brasileira de Botânica 2004; 27: 19-30. http://dx.doi.org/10.1590/ S0100-84042004000100003

Ferretti AR. Modelos de Plantio para a Restauração. In: Galvão APM, Medeiros ACS, editores. Restauração da Mata Atlântica em Áreas de sua Primitiva Ocorrência Natural. Colombo: Embrapa Florestas; 2002.
Galetti M. Os frugívoros da Santa Genebra. In: Morellato PC, Leitão-Filho HF. Ecologia e preservação de uma floresta tropical urbana. Campinas: UNICAMP; 1995. p. 66-69.

Gandolfi S, Leitão-Filho HF, Bezerra CLF. Levantamento florístico e caráter sucessional das espécies arbustivoarbóreas de uma floresta mesófila semidecídua no município de Guarulhos, SP. Revista Brasileira de Botânica 1995; 55(4):753-767.

Gibbs PE, Leitão-Filho HF. Floristic composition of an area of gallery forest near Moji-Guaçu, State of São Paulo, SP, Brazil. Revista Brasileira de Botânica 1978; 1: 151-156.

Herrera HAR, Rosot NC, Rosot MAD, Oliveira YMM. Análise florística e fitossociológica do componente arbóreo da Floresta Ombrófila Mista presente na reserva florestal EMBRAPA/EPAGRI, Caçador, SC - Brasil. Revista Floresta 2009; 39(3): 485-500.

Higuchi P, Silva AC, Ferreira TS, Souza ST, Gomes JP, Silva KM et al. Influência de variáveis ambientais sobre o padrão estrutural e florístico do componente arbóreo, em um fragmento de Floresta Ombrófila Mista Montana em Lages, SC. Ciência Florestal 2012; 22(1): 79-90. http://dx.doi.org/10.5902/198050985081

Holdridge LR, Grenke WC, Hatheway WH, Liang T, Tosi JA. Forest environment in tropical life zones: a pilot study. Pergamon Press; 1971. 747 p.

Instituto Brasileiro de Geografia e Estatística - IBGE. Manual técnico da vegetação brasileira. Departamento de Recursos Naturais e Estudos Ambientais; 1992.

Inoue MT, Roderjan CV, Kuniyoshi YS. Projeto Madeira do Paraná. Fundação de Pesquisas Florestais do Paraná; 1984.

Jarenkow JA, Waechter JL. Composição, estrutura e relações florísticas do componente arbóreo de uma floresta estacional no Rio Grande do Sul, Brasil. Revista Brasileira de Botânica 2001; 24(3): 263-272. http:// dx.doi.org/10.1590/S0100-84042001000300004

Klein RM. Aspectos dinâmicos da vegetação do sul do Brasil. Sellowia 1984; 36: 5-54.

Köeppen W. Climatologia: Con Un Estúdio de los Climas de La Tierra. Fondo de Cultura; 1948.

Lima TEO, Hosokawa RT, Machado SA, Klock U. Caracterização fitossociológica da vegetação no entorno de nascentes de um fragmento de Floresta Ombrofila Mista Montana na bacia do rio das Pedras, Guarapuava (PR). Ambiência 2012; 8(2): 229-244. http://dx.doi. org/10.5777/ambiencia.2012.02.01

Lorenzi H. Árvores Brasileiras: manual de identificação e cultivo de plantas arbóreas nativas do Brasil. Nova Odessa: Plantarum; 1998.

Loures L, Carvalho DA, Machado ELM, Marques JJGSM. Florística, estrutura e características do solo de 
um fragmento de floresta paludosa no sudeste do Brasil. Acta Botanica Brasilica, v. 21, p. 885-896, 2007. http:// dx.doi.org/10.1590/S0102-33062007000400013

Machado AM, Plácido AC,Bartoszeck S, Figueiredo Filho A, Oliveira EB. Dinâmica da distribuição diamétrica de bracatingais na região metropolitana de Curitiba. Revista Árvore 2006; 30(5): 759-768. http:// dx.doi.org/10.1590/S0100-67622006000500009

Martins FR. Estrutura de uma floresta mesófila. 2. ed. UNICAMP; 1993.

Martins-Ramos D, Chaves CL, Bortoluzzi RLC, Mantovani A. Florística de Floresta Ombrófila Mista Altomontana e de Campos em Urupema, Santa Catarina, Brasil. Revista Brasileira de Biociências 2011; 9(2): 156-166.

Metzger JP. Estrutura da paisagem e fragmentação: uma análise bibliográfica. Anais Academia Brasileira de Ciências 1999; 71(3): 445-463.

Motta FS, Beirsdorf MJC, Garcez RB. Zoneamento agrícola do Rio Grande do Sul e Santa Catarina: normas agro-climáticas. Ministério da Agricultura; 1971. 80 p.

Narvaes IS, Longhi SJ, BrenaDA. Florística e classificação da regeneração natural em Floresta Ombrófila Mista na Floresta Nacional de São Francisco de Paula, RS. Ciência Florestal 2008; 18(2): 233-245.

Nascimento ART, Longhi SJ, Brena DA. Estrutura e padrões de distribuição espacial de espécies arbóreas em uma amostra de Floresta Ombrófila Mista em Nova Prata, RS. Ciência Florestal 2001; 11(1): 105-119.

Negrelle RRB, Silva FC. Fitossociologia de um trecho de floresta com Araucaria angustifolia (Bert.) O. Kuntze no município de Caçador, SC. Boletim de Pesquisas Florestais 1992; 24-25: 37-54.

Neri AV, Soares MP, Meira-Neto JAA, Dias LE. Espécies de Cerrado com potencial para recuperação de áreas degradadas por mineração de ouro, Paracatu-MG. Revista Árvore 2011; 35(4): 907-918. http://dx.doi. org/10.1590/S0100-67622011000500016

Oliveira YMM, Rotta E. Área de distribuição natural de erva-mate. In: Anais do Seminário sobre atualidades e perspectivas florestais: Silvicultura da Erva-mate, 1983, Curitiba. Curitiba: EMBRAPA, Centro Nacional de Pesquisa de Florestas, 1985. 145 p. p. 17-36. (Documentos, n. 15).

Reis A, Kageyama PY. Restauração de áreas degradadas utilizando interações interespecíficas. In Kageyama PY, Oliveira RE, Moraes LFD, Engel VL, Gandara $\mathrm{FB}$, editors. Restauração ecológica de ecossistemas naturais. Fundação de Estudo e Pesquisas Agrícolas e Florestais; 2003. p. 91-110.
Reitz PR. Flora Ilustrada Catarinense. Itajaí; 1971.

Reitz R, Klein RM, Reis A. Projeto Madeira do Rio Grande do Sul. Sellowia 1983; 34-35: 1-525.

Reitz R. Euforbiáceas. In: Reitz R, editor. Flora Ilustrada Catarinense. Herbário Barbosa Rodrigues, Itajaí; 1988.

Rode R, Figueiredo-Filho A, Machado AS, Galvão F. Grupos florísticos e espécies discriminantes em povoamento de Araucaria angustifolia e uma Floresta Ombrófila Mista. Revista Árvore 2011; 35(2): 319-327. http://dx.doi.org/10.1590/S0100-67622011000200016

Rodrigues ER, Monteiro R, Cullen-Junior L. Dinâmica inicial da composição florística de uma área restaurada na região do Pontal do Paranapanema, São Paulo, Brasil. Revista Árvore 2010; 34(5): 853-861. http:// dx.doi.org/10.1590/S0100-67622010000500010

Rondon-Neto RM, Watzlawick LF, Caldeira MVW, Schoeninger ER. Análise florística e estrutural de um fragmento de Floresta Ombrófila Mista Montana, situado em Criúva, RS. Ciência Florestal 2002; 12(1): 29-37.

Sanquetta CR, Pizzatto W, Netto SP, Eisfeld RL, Figueiredo-Filho A. Estrutura vertical de um fragmento de Floresta Ombrófila Mista no Centro-Sul do Paraná. Revista Floresta 2002; 32(2): 267-276.

Siminski A, Fantini AC,Guries RP, Ruschel AR, Reis MS. Secondary Forest Succession in the Mata Atlantica, Brazil: Floristic and Phytosociological Trends. International Scholarly Research Network Ecology 2011; 2011: 1-19.

Sonego R,Backes A,Souza A. Descrição da estrutura de uma Floresta Ombrófila Mista, RS, Brasil, utilizando estimadores não-paramétricos de riqueza e rarefação de amostras. Acta Botanica Brasilica 2007; 21(4): 943-955. http://dx.doi.org/10.1590/S0102-33062007000400019

Sehnem A. Ciateáceas. In: Reitz R, editor. Flora Ilustrada Catarinense. Herbário Barbosa Rodrigues, Itajaí; n. CIAT; 1978.

Van Der Pijl L. Principles of dispersal in higher plants. 3rd ed. New York: Springer Verlag; 1982. http:// dx.doi.org/10.1007/978-3-642-87925-8

Viani RAG, Vieira AOS. Flora arbórea da bacia do rio Tibagi (Paraná, Brasil): Celastrales sensu Cronquist. Acta Botanica Brasilica 2007; 21(2): 457-472. http:// dx.doi.org/10.1590/S0102-33062007000200019

Vibrans AC, Sevegnani L,Uhlmann A, Schorn LA, Sobral MG, Gasper AL et al. Structure of mixed ombrophyllous forests with Araucaria angustifolia (Araucariaceae) under external stress in Southern Brazil. Revista de Biologia Tropical 2011; 59(3): 1371-1387. PMid:22017139. 